Pacific Journal of Mathematics

COBORDISM OF MANIFOLDS WITH STRONG ALMOST Bo 


\title{
COBORDISM OF MANIFOLDS WITH STRONG ALMOST TANGENT STRUCTURES
}

\author{
ROBERT GOGGINS
}

\begin{abstract}
This paper studies the unoriented cobordism classes of closed smooth manifolds whose tangent bundles admit nilpotent bundle endomorphisms.
\end{abstract}

1. Introduction. An almost tangent manifold is a smooth (differentiable of class $C^{\infty}$ ) manifold $M^{2 n}$ for which the structure group of its tangent bundle $\tau(M)$ reduces to the group of matrices of the form $\left(\begin{array}{ll}A & 0 \\ B & A\end{array}\right)$, $A \in \mathrm{GL}_{n}(R), B \in \operatorname{End}\left(R^{n}\right)$. The study of these manifolds is motivated by the observation that tangent manifolds have this property, i.e., if $N^{n}$ is a smooth manifold and $E^{2 n}$ is the total space of $\tau(N)$, then $E^{2 n}$ (a tangent manifold) is an almost tangent manifold.

Note that if $M^{2 n}$ is almost tangent, then the matrix

$$
\left(\begin{array}{cc}
0 & 0 \\
I_{n} & 0
\end{array}\right) \in \operatorname{End}\left(R^{2 n}\right)
$$

where $I_{n}$ is the $n \times n$ identity matrix, induces a bundle endomorphism $J$ : $\tau(M) \rightarrow \tau(M)$ having constant rank $n$ and satisfying $J^{2}=0$. As $J$ has constant rank, $\tau(M)$ is isomorphic to the Whitney sum im $J \oplus \operatorname{ker} J$, and $\operatorname{im} J=\operatorname{ker} J$, so one has $\tau(M) \cong 2(\operatorname{im} J)$. Thus $M^{2 n}$ has an almost complex structure and, for $M^{2 n}$ closed, one is led to the well-known result that $M$ is cobordant to a square $N^{n} \times N^{n}$. In particular, the cobordism class of $M$ is decomposable in $\mathfrak{N}_{2 n}$, the unoriented cobordism group.

In [4] Epiopoulous defines an almost tangent structure of order $r$ on a smooth manifold $M^{n}$ to be a bundle endomorphism $J: \tau(M) \rightarrow \tau(M)$ of constant rank which satisfies $J^{r} \neq 0$ and $J^{r+1}=0$ (thus, an almost tangent manifold has an almost tangent structure of order 1). Motivated by the splitting property enjoyed by almost tangent manifolds, one says that an almost tangent structure of order $r, J$, on $M^{n}$ is strong and of type $k$ provided $J^{r}: \tau(M) \rightarrow \tau(M)$ has constant rank $k$.

Proposition 1.1. Let $r, k \geq 1$, and $n \geq(r+1) k$. A compact smooth manifold $M^{n}$ has a strnog almost tangent structure of order $r$ and type $k$ iff

$$
\tau(M) \cong(r+1) \xi^{k} \oplus \eta^{n-(r+1) k}
$$


for some vector bundles $\xi^{k}, \eta^{n-(r+1) k} \rightarrow M^{n}$. (Note: $(r+1) \xi^{k}$ denotes the $(r+1)$-fold Whitney sum of $\xi^{k}$.)

Proof. If $J$ is such a structure, then for each fiber $T M_{x}$ of $\tau(M)$, one has $T M_{x} \cong(r+1)\left(\operatorname{im} J_{x}^{r}\right) \oplus V_{x}$, where $J_{x}=J \mid T M_{x}, \oplus$ is the direct sum of vector spaces, and $V_{x}$ is a complementary vector subspace of $T M_{x}$. Since $J^{r}$ has constant rank $k$, necessity follows by setting $\xi^{k}=\operatorname{im} J^{r}$. For sufficiency, note that if $\tau(M) \cong(r+1) \xi^{k} \oplus \eta$, then $J: \tau(M) \rightarrow \tau(M)$, defined by $J\left(x_{1}, \ldots, x_{r+1}, y\right)=\left(0, x_{1}, \ldots, x_{r}, 0\right)$, has the desired properties.

This splitting characterization will be exploited to study the unoriented cobordism classes of closed smooth manifolds having strong almost tangent structures. Preliminary results are presented in $\$ 2$. Manifolds with structures of type 1 are studied in $\$ 3$, and manifolds with structures of type $k, k>1$, are treated briefly in $\S 4$. Most of the results of this paper are contained in the author's dissertation at the University of Virginia.

2. Preliminaries. In [8] Stong shows that if $\tau(M)$ contains an odd-dimensional subbundle, then $w_{n}(M)[M]=0$. The following lemma, which is used throughout the paper, is a direct consequence.

LEMMA 2.1. If $M^{n}$ has a strong almost tangent structure of type $k$, and $k$ is odd, then $w_{n}(M)[M]=0$.

The extreme case of a strong almost tangent structure on $M^{n}, n \geq 2$, is a structure of order $(n-1)$ and type 1, i.e., $\tau\left(M^{n}\right) \cong n \xi^{1}$. If $w(\xi)=$ $1+a$, then $w(M)=(1+a)^{n}$ and $w_{i}(M)=\left(\begin{array}{l}n \\ l\end{array}\right) a^{l}$. By 2.1, $a^{n}[M]=$ $w_{n}(M)[M]=0$. Since all Stiefel-Whitney numbers of $M$ are multiples of $a^{n}[M]=0$, it follows that $M^{n}$ bounds.

The converse of this result is false, for in [6] Rosendo and Gadea show that for $n \neq 3$ or 7 , the sphere $S^{n}$ does not admit an almost tangent structure of order $(n-1)$. However, if $M^{n}$ bounds, then $M$ is cobordant to an $n$-fold product of circles which has tangent bundle isomorphic to an $n$-fold Whitney sum of trivial line bundles. Thus, one has

Proposition 2.2. A class $\alpha \in \mathfrak{N}_{n}, n \geq 2$, is represented by a manifold with a strong almost tangent structure of order $(n-1)$ and type 1 iff $\alpha=0$.

THEOREM 2.3. Every 3-manifold $M^{3}$ has $\tau(M) \cong 3 \xi^{1}$ for some $\xi^{1} \rightarrow M^{3}$ (hence, every $M^{3}$ has a strong almost tangent structure of order 2 and type 1). 
Proof. By restricting attention to components, one may assume $M^{3}$ connected. If $v=1+v_{1}$ is the Wu class, then

$$
w(M)=\operatorname{Sq}\left(1+v_{1}\right)=1+v_{1}+v_{1}^{2}=1+w_{1}+w_{1}^{2} .
$$

Let $\xi^{1}$ be the determinant of $\tau\left(M^{3}\right)$. Then

$$
w(\xi \otimes \tau(M))=\left(1+w_{1}(\xi)\right)^{3}+w_{1}\left(1+w_{1}(\xi)\right)^{2}+w_{1}^{2}\left(1+w_{1}(\xi)\right),
$$

and since $w_{1}(\xi)=w_{1}$, it follows that $w(\xi \otimes \tau(M))=1$, so $\xi \otimes \tau(M)$ is a $\operatorname{Spin}_{3}$ bundle, and there is a lift:

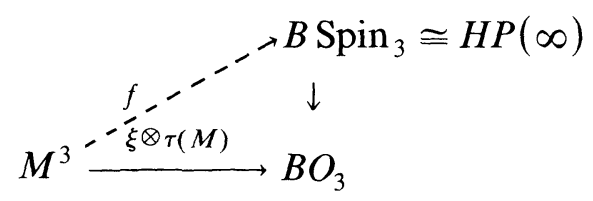

Since $H P(\infty)$ is 3-connected, $f$ is null homotopic, so $\xi \otimes \tau(M)$ is trivial. Therefore,

$$
\tau(M) \cong 1 \otimes \tau(M) \cong(\xi \otimes \xi) \otimes \tau(M) \cong \xi \otimes 3 \cong 3 \xi^{1} .
$$

REMARKs. (1) Clark and Goel [2] prove this for $M^{3}=S^{3}$.

(2) This is a modification of the well-known argument that an orientable 3-manifold is parallelizable.

Fix integers $r \geq 2, k \geq 1$, and, for $n \geq r k$, define $A_{n}(r, k)$ to be the subset of classes in $\mathfrak{N}_{n}$ represented by manifolds having strong almost tangent structures of order $(r-1)$ and type $k$. In terms of 1.1,

$$
A_{n}(r, k)=\left\{\alpha \in \mathfrak{R}_{n} \mid \exists M^{n} \in \alpha \text { with } \tau(M) \cong r \xi^{k} \oplus \eta\right\} .
$$

It is easily seen that $A_{n}(r, k)$ is a subgroup of $\mathfrak{R}_{n}$, and if $A(r, k)$ is the weak direct sum $\Sigma_{n \geq r k} A_{n}(r, k)$, then it also follows that $A(r, k)$ is an algebra ideal of $\mathfrak{N}_{*}$. Most of this paper is devoted to the study of the ideals $A(r, 1)$. For convenience, we will write $A(r)$ for $A(r, 1)$.

3. The ideals $A(r)$. In this section some indecomposable classes of $A(r)$ are exhibited. Recall the manifolds $R P\left(n_{1}, \ldots, n_{t}\right)$ as defined in [7]. $R P\left(n_{1}, \ldots, n_{t}\right)$ is the real projective space bundle of $\lambda_{1} \oplus \cdots \oplus \lambda_{t} \rightarrow$ $R P\left(n_{1}\right) \times \cdots \times R P\left(n_{t}\right)$, where $\lambda_{l}$ is the pullback of the canonical line bundle over the $i$ th factor, and has dimension $n_{1}+\cdots+n_{t}+t-1$. In [7] Stong proves that for $t>1, R P\left(n_{1}, \ldots, n_{t}\right)$ is indecomposable iff

$$
\sum_{i=1}^{t}\left(\begin{array}{c}
n_{1}+\cdots+n_{t}+t-2 \\
n_{l}
\end{array}\right)
$$

is odd. 
THEOREM 3.1. There exist indecomposable classes $\alpha_{n} \in A_{n}(r)$ for:

(a) $n=4 k, r=4 k-2(k \geq 1)$,

(b) $n=4 k+1, r=4 k-1(k \geq 1)$,

(c) $n=4 k+2, r=4 k-1(k \geq 1)$, and

(d) $n=2^{p}(2 q+1)-1, r=2^{p+1} q-1(p \geq 2, q \geq 1)$.

Proof. The indecomposability of the classes to be defined follows from the above remark. For $R P\left(n_{1}, \ldots, n_{t}\right)$, let $p$ be the projection $R P\left(n_{1}, \ldots, n_{t}\right) \rightarrow R P\left(n_{1}\right) \times \cdots \times R P\left(n_{t}\right)$, let $\gamma$ be the canonical line bundle over $R P\left(n_{1}, \ldots, n_{t}\right)$ consisting of pairs

(line in a fiber of $\lambda_{1} \oplus \cdots \oplus \lambda_{t}$, vector in that line),

and let $\mu^{t-1}$ be the bundle along the fibers so that

$$
\tau\left(R P\left(n_{1}, \ldots, n_{t}\right)\right) \cong p^{*} \tau\left(R P\left(n_{1}\right) \times \cdots \times R P\left(n_{t}\right)\right) \oplus \mu^{t-1} .
$$

(a) Let $\alpha_{4 k}$ be the class of $R P(4 k-3,1,0)$ which has $\tau$ isomorphic to $(4 k-2) p^{*} \lambda_{1} \oplus \mu^{2}$.

(b) Let $\alpha_{4 k+1}$ be the class of $R P(4 k-2,1,0)$ which has $\tau$ isomorphic to $(4 k-1) p^{*} \lambda_{1} \oplus \mu^{2}$.

(c) Let $\alpha_{4 k+2}$ be the class of $R P(1,1,0, \ldots, 0)$ with $(4 k-1)$ zeros. This manifold has $\tau$ isomorphic to $1 \oplus 1 \oplus \mu^{4 k}$, and it is well known that

$$
\begin{aligned}
1 \oplus \mu^{4 k} & \cong \gamma \otimes p^{*}\left(\lambda_{1} \oplus \cdots \oplus \lambda_{4 k+1}\right) \\
& \cong\left(\gamma \otimes p^{*} \lambda_{1}\right) \oplus \cdots \oplus\left(\gamma \otimes p^{*} \lambda_{4 k+1}\right)
\end{aligned}
$$

For $i=3, \ldots, 4 k+1, p^{*} \lambda_{i}$ is trivial (pullback of canonical bundle over $R P(0)=$ point $)$. Thus, it follows that

$$
\begin{aligned}
\tau(R P(1,1,0, \ldots, 0)) & \cong 1 \oplus\left(\gamma \otimes p^{*} \lambda_{1}\right) \oplus\left(\gamma \otimes p^{*} \lambda_{2}\right) \oplus(4 k-1) \gamma \\
& \cong(4 k-1) \gamma \oplus \eta^{3} .
\end{aligned}
$$

(d) Let $\alpha_{2^{p}(2 q+1)-1}$ be the class of $R P\left(2^{p+1} q-2,1,0, \ldots, 0\right)$ with $\left(2^{p}-1\right)$ zeros. This manifold has $\tau$ isomorphic to $\left(2^{p+1} q-1\right) p^{*} \lambda_{1} \oplus$ $\mu^{2^{p}}$.

The question as to whether these are the largest values of $r$ for which there is an indecomposable $\alpha_{n} \in A_{n}(r)$ is answered partially by

THEOREM 3.2. Suppose $M^{n}$ is a closed manifold. (a) If $\tau\left(M^{n}\right) \cong$ $(n-1) \xi^{1} \oplus \eta^{1}$, then $M^{n}$ bounds. (b) If $n \equiv 2 \bmod 4, n \geq 6$, and $\tau\left(M^{n}\right) \cong$ $(n-2) \xi^{1} \oplus \eta^{2}$, then $M^{n}$ bounds. 
ReMARKs. (1) This theorem generalizes 2.2 in dimensions $\not \geq 0 \bmod 4$.

(2) The result shows that the results of 3.1(a)-(c) are best possible. In fact, stronger results are obtained. Namely, the values of $r$ in 3.1(a)-(c) are the largest for which there is a nonzero $\alpha_{n} \in A_{n}(r)$. (3) An example showing that $3.1(\mathrm{~d})$ is not best possible will be presented later in this section.

To begin to establish 3.2(a), suppose $\tau\left(M^{n}\right) \cong(n-1) \xi^{1} \oplus \eta^{1}$ and consider $\left[M^{n}, \xi, \eta\right]$ as an element of $\mathfrak{R}_{n}(R P(\infty) \times R P(\infty))$. The result is trivial for $n=1$, so assume $n \geq 2$. Consider $X^{n}=R P\left(\lambda^{1} \oplus(n-1)\right) \rightarrow$ $R P(1)$ where $\lambda^{1}$ is the canonical line bundle over $R P(1)$. Let $\gamma$ be the canonical line bundle over $X^{n}$, and also denote by $\lambda^{1}$ the pullback of $\lambda^{1}$ to $X^{n}$ by the projection. Then one has $\tau(X) \cong(n-1) \gamma \oplus(\gamma \otimes \lambda)$, and there is the element $\left[X^{n}, \gamma, \gamma \otimes \lambda\right] \in \mathfrak{N}_{n}(R P(\infty) \times R P(\infty))$. If $c=w_{1}(\gamma)$ and $d=w_{1}(\gamma \otimes \lambda)$, then it is a routine calculation to show that for $0 \leq i \leq n, c^{i} d^{n-i}[X]=1$ iff $i \equiv \bmod 2$.

LEMMA 3.3. If $\left[M^{n}, \xi, \eta\right] \neq 0$, then $\left[M^{n}, \xi, \eta\right]=\left[X^{n}, \gamma, \gamma \otimes \lambda\right]$.

Proof. Suppose $\left[M^{n}, \xi, \eta\right] \neq 0$ and let $w(\xi)=1+a, w(\eta)=1+b$. By the remarks above, it suffices to show that for $0 \leq i \leq n, a^{i} b^{n-i}\left[M^{n}\right]$ $=1$ iff $i \equiv n \bmod 2$.

For $n=2$, one has $\tau\left(M^{2}\right) \cong \xi^{1} \oplus \eta^{1}, w(M)=(1+a)(1+b)=1+$ $(a+b)+a b$, and $a b[M]=w_{2}[M]=0$ (by 2.1), so $M^{2}$ bounds. Therefore, $w_{1}^{2}[M]=0$ which implies $a^{2}[M]=b^{2}[M]=1$, so the result is true for $n=2$.

Having assumed inductively the result true for $2 \leq n^{\prime}<n$, suppose one has $\left[M^{n}, \xi, \eta\right] \neq 0$. Now observe that $\left[M^{n}, \xi, \eta\right]$ must have a nonzero characteristic number divisible by $a$. (To see this, note that if $n$ is odd, the $w_{1}(\xi)=b$, so $b^{n}[M]=w_{1} b^{n-1}[M]=\operatorname{Sq}^{1} b^{n-1}[M]=0$. If $n$ is even, say $n=2^{p}(2 q+1)(p>0, q \geq 0)$, one sees that

$$
v_{2^{p}}(M)=a^{2^{p}}+b^{2^{p}}+a^{2^{p}-1} b, \text { so } b^{2^{p}}=v_{2^{p}}(M)+a^{2^{p}}+a^{2^{p}-1} b .
$$

Hence,

$$
\begin{aligned}
b^{n}[M] & =\left\{v_{2^{p}}(M)+a^{2^{p}}+a^{2^{p}-1} b\right\} b^{2^{p+1}} q[M] \\
& \left.=\operatorname{Sq}^{2^{p}} b^{2^{p+1}} q M\right]+a x[M]
\end{aligned}
$$


and

$$
\left.\mathrm{Sq}^{2^{p}} b^{2^{p+1}} q[M]=\left(\begin{array}{c}
2^{p+1} q \\
2^{p}
\end{array}\right) b^{n}[M]=0 .\right)
$$

Dualizing $\xi$ in $M$ gives a submanifold $N^{n-1} \hookrightarrow M$ with

$$
\tau(N) \cong(n-2)(\xi \mid N) \oplus(\eta \mid N), \text { and } \operatorname{ax}[M]=(x \mid N)[N] .
$$

Since $[M, \xi, \eta]$ has a nonzero number divisible by $a,[N,(\xi \mid N),(\eta \mid N)] \neq$ 0 . The result follows by applying the induction hypothesis to $\left[N^{n-1}\right.$, $(\xi \mid N),(\eta \mid N)]$.

The result of 3.2(a) now follows, for if $\left[M^{n}, \xi, \eta\right]=0$, then $M^{n}$ bounds, and if $\left[M^{n}, \xi, \eta\right]=\left[X^{n}, \gamma, \gamma \otimes \lambda\right]$, then $M$ is cobordant to $X$, and Conner and Floyd have shown [3] that $X$ bounds.

The result of 3.2(b) is aided by the notion of a Yoshida manifold. As in [10] and [11], one defines a Yoshida manifold to be a closed manifold $M^{n}$ which has Stiefel-Whitney numbers satisfying

$$
w_{\omega}(M)[M]=0 \quad \text { if } \omega \neq\left(2^{p_{1}}, 2^{p_{2}}, \ldots, 2^{p_{t}}\right) .
$$

Stong shows in [10] that if $M^{n}$ is a Yoshida manifold, and $n$ is not a power of 2 , then $M^{n}$ bounds.

Proof of 3.2(b). Let $n=4 k+2, k \geq 1$, and suppose $\tau\left(M^{4 k+2}\right) \cong$ $4 k \xi^{1} \oplus \eta^{2}$. It is claimed that the element

$$
\left[M^{4 k+2}, 4 \xi\right] \in \mathfrak{R}_{4 k+2}(H P(\infty))
$$

is zero (it would then follow that $M$ bounds). Let $w(\xi)=1+a, w(\eta)=1$ $+b_{1}+b_{2}$, and note that $[M, 4 \xi]$ is determined by characteristic numbers $a^{4 j} w_{\omega}(M)[M]$. For $k=1$, one has $w\left(M^{6}\right)=(1+a)^{4}\left(1+b_{1}+b_{2}\right)$ and $a^{4}=w_{4}$, so to establish $\left[M^{6}, 4 \xi\right]=0$, it suffices to show $M^{6}$ bounds. Now $w_{6}[M]=0($ by 2.1$)$, and $w_{3}^{2}[M]=0$ since $w_{3}=0$. Also,

$$
\begin{aligned}
w_{1}^{6}[M] & =b_{1}^{6}[M]=\mathrm{Sq}^{3} b_{1}^{3}[M]=v_{3} b_{1}^{3}[M]=b_{1} b_{2} b_{1}^{3}[M] \\
& =b_{1}^{2} \mathrm{Sq}^{1} b_{1} b_{2}[M]=b_{1}^{2} \mathrm{Sq}^{1} \mathrm{Sq}^{1} b_{2}[M]=0
\end{aligned}
$$

since $\mathrm{Sq}^{1} \mathrm{Sq}^{1}=0$. Since $w_{6}, w_{3}^{2}$ and $w_{1}^{6}$ determine on $\mathfrak{R}_{6}$, it follows that $M^{6}$ bounds.

Assume the result true for $1 \leq k^{\prime}<k$ and that one has $\left[M^{4 k+2}, 4 \xi\right]$. Dualizing $4 \xi$ in $M$ gives $N^{4 k-2} \hookrightarrow M$ with $\tau(N) \cong 4(k-1)(\xi \mid N) \oplus$ $(\eta \mid N)$. Then $[N, 4(\xi \mid N)]=0$ by induction, so $j>0$ implies

$$
a^{4 j} w_{\omega}(M)[M]=\left\{a^{4 j-4} w_{\omega}(M) \mid N\right\}[N]=0 .
$$


Therefore, all numbers involving $a^{4}$ vanish, so $w(M)$ is equivalent in numbers to $1+w_{1}+w_{2}$, i.e., $M$ is a Yoshida manifold, and for $k \geq 1$, $4 k+2$ cannot be a power of 2 . Thus, $M$ bounds, so the remaining numbers $w_{\omega}(M)[M]$ are zero. Therefore, $\left[M^{4 k+2}, 4 \xi\right]=0$.

It will now be shown that 3.1(d) is not best possible. Consider the indecomposable manifold $R P(7,14,0) \rightarrow R P(7) \times R P(14)$ and pull the fibration back over $R P(7) \times P^{8} \times P^{4} \times P^{2}$ by a degree 1 map $f$, where

$$
P^{8}=R P(\lambda \oplus 1) \rightarrow R P(7), \quad P^{4}=R P(\lambda \oplus 1) \rightarrow R P(3),
$$

and

$$
P^{2}=R P(\lambda \oplus 1) \rightarrow R P(1) .
$$

( $f$ is the identity on the first factor, and on $P^{8} \times P^{4} \times P^{2}, f$ is the map which classifies the tensor product of the bundles along the fibers $\left.P^{8} \times P^{4} \times P^{2} \times \stackrel{\mu_{1}^{1} \otimes \mu_{2}^{1} \otimes \mu_{3}^{1}}{\rightarrow} R P(14)\right)$. By Proposition 2.4 of [7], $M^{23}=$ $f^{*}\{R P(7,14,0)\}$ is indecomposable. Moreover, $M^{23}$ fibers over $R P(7) \times$ $R P(7) \times R P(3) \times R P(1)$, so $\tau(M)$ has 18 linearly independent sections. Therefore, $\tau(M) \cong 18 \oplus \eta^{5}$.

Since this construction uses the fact that $\tau(R P(m))$ is trivial for $m=1,3$ and 7 , it cannot be generalized to produce similar examples in other dimensions. Nonbounding examples (which are decomposable) are easily constructed in dimensions $4 k+3, k \geq 1$, by taking a product of a nonbounding $P^{5}$ with $R P(4 k-2)$. The product then has $\tau$ isomorphic to $(4 k-1) \lambda^{1} \oplus \eta^{4}\left(\tau\left(P^{5}\right)\right.$ has a section). It will be shown momentarily that this result is best possible.

This discussion raises two questions: (1) Are there other examples of indecomposables in $A_{n}(r)$ with $n=2^{p}\left(2_{q}+1\right)-1$ and $r>2^{p+1} q-1$ ? (2) What is the largest value of $r$ for which there exist indecomposables in $A_{n}(r), n=2^{p}(2 q+1)-1(p>2, q>0)$ ? Theorem $3.1(\mathrm{~d})$ is best possible for $p=2$ by

THEOREM 3.4. If the closed manifold $M^{4 k+3}, k \geq 0$, has $\tau(M) \cong 4 k \xi^{1}$ $\oplus \eta^{3}$, then $M$ bounds.

LEMMA 3.5 (a) If $\tau\left(M^{7}\right) \cong 4 \xi^{1} \oplus \eta^{3}$, then $M^{7}$ bounds. (b) If $\tau\left(M^{11}\right) \cong$ $8 \xi^{1} \oplus \eta^{3}$, then $M^{11}$ bounds. 
Proof. (a) A nonbounding $P^{7}$ must have $w_{5}(P) w_{2}(P)[P] \neq 0$. If $\tau\left(M^{7}\right) \cong 4 \xi^{1} \oplus \eta^{3}$, then $w_{5}(M)=w_{1}^{4}(\xi) w_{1}(\eta), w_{2}(M)=w_{2}(\eta)$, so

$$
\begin{aligned}
w_{5}(M) w_{2}(M)[M] & =w_{1}(\eta) w_{2}(\eta) w_{1}^{4}(\xi)[M]=w_{1}(M) w_{2}(M) w_{1}^{4}(\xi)[M] \\
& =\operatorname{Sq}^{3} w_{1}^{4}(\xi)[M]=0 .
\end{aligned}
$$

(b) Assuming $\tau\left(M^{11}\right) \cong 8 \xi^{1} \oplus \eta^{3}$, let

$$
\begin{gathered}
w(\xi)=1+a \\
w(\eta)=1+w_{1}+w_{2}+w_{3}=1+w_{1}(M)+w_{2}(M)+w_{3}(M) .
\end{gathered}
$$

Then $a^{8} w_{3}[M]=0$ by $2.1, a^{8} w_{1}^{3}[M]=\operatorname{Sq}^{1} a^{8} w_{1}^{2}[M]=0$, and $a^{8} w_{1} w_{2}[M]$ $=\mathrm{Sq}^{3} a^{8}[M]=0$, so $w(M)$ is equivalent in numbers to $1+w_{1}+w_{2}+w_{3}$. The proof that all $w_{\omega}[M]$ involving $w_{1}, w_{2}$ and $w_{3}$ are zero is routine, but tedious, and will be omitted.

Proposition 3.6. If $M^{4 k+3}, k \geq 0$, has $w(M)$ equivalent to $1+w_{1}+$ $w_{2}+w_{3}$ in numbers, then $M$ bounds.

Proof. Remarks in the proof of 3.5 give a basis for induction on $k$, so assume the result true for all $2 \leq k^{\prime}<k$, and suppose $w\left(M^{4 k+3}\right) \sim 1+$ $w_{1}+w_{2}+w_{3}$. Note that if $M$ is nonbounding, then some Stiefel-Whitney number divisible by $w_{3}$ is nonzero (otherwise $M$ is a Yoshida manifold with $4 k+3=2^{s}$ impossible).

Suppose $w_{3}^{2} w_{\omega}[M] \neq 0$. Dualize $w_{3}^{2}$ in $M$ to get $N^{4 k-3} \hookrightarrow M$ with $\bar{w}(N) \sim 1+w_{1}+w_{2}+w_{3}$ (use of restriction homomorphisms is omitted in this proof) and $w_{3}^{2} w_{\omega}[M]=\bar{w}_{\omega}[N]$. By Proposition 5 of [9], $N$ is cobordant to a Dold manifold $P\left(2^{r}-1,2^{s+1}-2\right), 0<r \leq s$. Then $4 k-$ $3=2^{r}-1+2^{s+2}-4$, so $r=1$ and $4 k+3=2^{s+2}+3$ with $N^{2^{s+2}-3} \sim$ $P\left(1,2^{s+1}-2\right)$ having $\bar{w}=1+d+c d, d \in H^{2}\left(P\left(1,2^{s+1}-2\right) ; Z_{2}\right), c \in$ $H^{1}\left(P\left(1,2^{s+1}-2\right) ; Z_{2}\right)$. The only nonzero number of $N$ is then $\bar{w}_{3} \bar{w}_{2}^{2+1}-3[N]$, so the only possible nonzero number of $M$ involving $w_{3}^{2}$ is $w_{3}^{3} w_{2}^{2^{s+1}-3}[M]$.

Consider $M \stackrel{2^{s} \tau}{\rightarrow} B O$ and pull back to $B O_{3-2} s$ so that $w \sim 1+w_{1}^{2^{s}}+$ $w_{2}^{2^{s}}+w_{3}^{2^{s}}$. Now $w_{3}^{2^{s}} w_{\omega}[M]=0$ (note that $s>1$ since $k>2$ ), so one can dualize $w_{2}^{2^{s}}$, which gives $N^{2^{s+1}+3} \hookrightarrow M$ with

$$
w(N) \sim\left(1+w_{1}+w_{2}+w_{3}\right) /\left(1+w_{1}^{2^{s}}+w_{2}^{2^{s}}\right),
$$


and $w_{2}^{2^{s}} w_{\omega}[M]=w_{\omega}[N]$. Now $w_{2}^{2^{s}}$ contributes zero on $N$, for $w_{2}^{2^{s}} x_{3}[N]=$ $w_{2}^{2^{s+1}} x_{3}[M]$, and

$$
w_{2}^{2^{s+1}} w_{1}^{3}[M]=\operatorname{Sq}^{1}\left(w_{2}^{2^{s+1}} w_{1}^{2}\right)[M]=0,
$$

$$
\begin{aligned}
& w_{2}^{2^{s+1}} w_{1} w_{2}[M]=\mathrm{Sq}^{3} w_{2}^{2^{s+1}}[M]=0, \text { and } \\
& w_{2}^{2^{s+1}} w_{3}[M]=w_{2}^{2^{s+1}}\left(w_{1} w_{2}+\mathrm{Sq}^{1} w_{1} w_{2}\right)[M]=2 w_{1} w_{2}^{2^{s+1}+1}[M]=0 .
\end{aligned}
$$

Therefore, $w(N) \sim\left(1+w_{1}+w_{2}+w_{3}\right) /\left(1+w_{1}^{2^{s}}\right)$. Dualize $w_{1}^{2^{s}}$ in $N$ to get $P^{2^{s}+3} \hookrightarrow N$ with $w(P) \sim\left(1+w_{1}+w_{2}+w_{3}\right) /\left(1+w_{1}^{2^{s+1}}\right)$. By dimension, $w_{1}^{2^{s+1}}=0$ in $H^{*}(P)$, so $w(P) \sim 1+w_{1}+w_{2}+w_{3}$, and $P$ bounds by induction. Therefore, $w_{1}^{2^{s}} w_{\omega}[N]=w_{\omega}[P]=0$, i.e., $w_{1}^{2^{s}}$ contributes zero on $N$, so $w(N) \sim 1+w_{1}+w_{2}+w_{3}$.

Hence, $N$ bounds by induction, so $w_{3}^{3} w_{2}^{2^{s+1}-3}[M]=w_{3}^{3} w_{2}^{2^{s}-3}[N]=0$. Therefore, all numbers of $M$ involving $w_{3}^{2}$ are zero.

Now dualize $w_{3}$ in $M$ to get $P^{4 k} \hookrightarrow M$ with $P \rightarrow B O$ reducing to $B O_{2}$, so one can dualize $w_{2}$ in $P$ to get $N^{4 k-2} \hookrightarrow P \hookrightarrow M$ with $\bar{w}(N) \sim 1+w_{1}+$ $w_{2}$ and $w_{2} w_{3} w_{\omega}[M]=\bar{w}_{\omega}[N]$. By Proposition $3^{\prime}$ of [9], $N$ is cobordant to $R P\left(2^{p+1}-2\right) \times R P\left(2^{q+1}-2\right), 0 \leq p \leq q$. Then $4 k-2=2^{p+1}+2^{q+1}$ -4 implies $p=0$, so $N^{2^{q+1}-2} \sim R P\left(2^{q+1}-2\right)$ having only $\bar{w}_{1}^{2^{q+1}-2}[N]$ nonzero. Therefore $w_{2} w_{3} w_{1}^{2^{q+1}-2}[M]$ is the only possible nonzero number involving $w_{2} w_{3}$.

Dualize $w_{1}^{2^{q}}$ in $M$ to get $Q^{2^{q}+3}$ with

$$
w(Q) \sim\left(1+w_{1}+w_{2}+w_{3}\right) /\left(1+w_{1}^{2^{q}}\right) .
$$

But $w_{1}^{2^{q}}$ contributes zero on $Q$, for $w_{1}^{2^{q}} x_{3}[Q]=w_{1}^{2^{q}} x_{3}[M]$ (see 3.7). Therefore $w(Q) \sim 1+w_{1}+w_{2}+w_{3}$, so $Q$ bounds by induction. Hence,

$$
w_{2} w_{3} w_{1}^{2^{q+1}-1}[M]=w_{2} w_{3} w_{1}^{2^{q}-1}[Q]=0 .
$$

Hence, the only possible nonzero number of $M$ divisible by $w_{3}$ is

$$
w_{1}^{4 k} w_{3}[M]=w_{1}^{4 k}\left(w_{1} w_{2}+\mathrm{Sq}^{1} w_{2}\right)[M]=2 w_{1}^{4 k+1} w_{2}[M]=0 .
$$

Therefore, $M$ bounds.

Proof of 3.4. Lemma 3.5 gives a basis for induction on $k$, so assume the result true for $1 \leq k^{\prime}<k$, and suppose $\tau\left(M^{4 k+3}\right) \cong 4 k \xi^{1} \oplus \eta^{3}$. Let $a=w_{1}(\xi)$ and note that any $w_{\omega}$ divisible by $a$ can be written $w_{\omega}=a^{4 s} w_{\omega^{\prime}}$, $0<s \leq k$, where $w_{\omega^{\prime}}$ is a polynomial in $w(\eta)$. Dualizing $4 s \xi$ in $M$ gives $N^{4(k-s)+3} \hookrightarrow M$ with $\tau(N) \cong 4(k-s)(\xi \mid N) \oplus(\eta \mid N)$. If $s<k$, then $N$ bounds by induction; if $s=k$, then $\operatorname{dim} N=3$ and $N$ bounds. Therefore, $a^{4 s} w_{\omega^{\prime}}[M]=w_{\omega^{\prime}}(N)[M]=0$. Hence, all numbers divisible by $a$ are zero, so $w(M) \sim w(\eta)$ in numbers. The result follows by 3.6. 
4. The ideals $A(r, k), k>1$. Here the case $k>1$ is treated briefly; results are not as extensive as those of $\S 3$. By 3.1, one has

COROLlaRY 4.1. Let $k \geq 1$. There exist indecomposables $\alpha_{n} \in A_{n}(r, k)$ for:

(a) $n=4 m, r=[(4 m-2) / k](m \geq 1)$

(b) $n=4 m+1, r=[(4 m-1) / k](m \geq 1)$

(c) $n=4 m+2, r=[(4 m-1) / k](m \geq 1)$, and

(d) $n=2^{p}(2 q+1)-1, r=\left[\left(2^{p+1} q-1\right) / k\right](p \geq 2, q \geq 1)$.

provided that $r \geq 2$ in each case $([x]$ is the greatest integer $\leq x)$.

There is a subalgebra $E=\Sigma_{k \geq 0} E_{2 k}$ of $\mathfrak{N}_{*}$, where $E_{2 k}$ is the subset of classes of $\mathfrak{R}_{2 k}$, represented by weakly almost complex manifolds for which all Chern numbers divisible by an odd-dimensional Chern class are zero. Floyd [5] showed there are generators $x_{i}, i>1, i \neq 2^{t}-1$, of $\mathfrak{N}_{*}$ such that

$$
E \subset Z_{2}\left[x_{\imath}^{\varepsilon_{l}} \mid \varepsilon_{2 k-1}=8, \varepsilon_{2^{k}}=8, \varepsilon_{2^{k} k(2 \jmath+1)}=4\right] .
$$

(It was later shown by Buhštaber [1] that equality holds in 4.2.)

If $M^{r k+\jmath}(r \geq 2)$ has $\tau(M) \cong r \xi^{k} \oplus \eta^{j}$, then dualizing $(r-2) \xi^{k} \oplus \eta^{j}$ in $M$ gives $N^{2 k} \hookrightarrow M$ with $\tau(N) \cong 2\left(\xi^{k} \mid N\right)$. Then $w_{r k+j}(M)[M]=$ $w_{2 k}(N)[N]$, and since $\tau(N) \cong 2(\xi \mid N)$, it follows that $[N] \in E_{2 k}$. By 4.2 one sees that $E_{2 k}=0$ for $0<2 k<16$ and for $2 k \neq 0 \bmod 8$. Thus, one has the following generalization of Proposition 2.1:

PROPOSITION 4.3. Suppose $M^{n}$ has a strong almost tangent structure of type $k$. If $k=4$ or if $k \neq 0 \bmod 4$, then $w_{n}(M)[M]=0$.

Using this, it is easy to show that for $k=2,4.1$ (a) is best possible. Also, for $k=2,4.1(\mathrm{~b})$ is best possible:

Proposition 4.4. (a) If $M^{4 m}, m>0$, is closed and $\tau(M) \cong 2 m \xi^{2}$, then $M$ bounds. (b) If $M^{4 m+1}, m>0$, is closed and $\tau(M) \cong 2 m \xi^{2} \oplus \eta^{1}$, then $M$ bounds.

Proof. (a) Let $w(\xi)=1+a_{1}+a_{2}$ so $w(M)=\left(1+a_{1}^{2}+a_{2}^{2}\right)^{m}$. Then all Stiefel-Whitney numbers of $M$ are sums of terms $a_{1}^{2 j} a_{2}^{2 j}[M], 2 i+4 j$ $=4 m$. For $i>0$,

$$
a_{1}^{2 i} a_{2}^{2 j}[M]=\operatorname{Sq}^{1}\left(a_{1}^{2 i-1} a_{2}^{2 j}\right)[M]=w_{1} a_{1}^{2 l-1} a_{2}^{2 J}[M]=0
$$


since $w_{1}=0$, and for $i=0$, one has $a_{2}^{2 m}[M]=w_{4 m}[M]=0$ by 4.3. For (b), let $w(\xi)=1+a_{1}+a_{2}, w(\eta)=1+b$ so

$$
w(M)=\left(1+a_{1}^{2}+a_{2}^{2}\right)^{m}(1+b) .
$$

Then all Stiefel-Whitney numbers of $M$ are sums of terms $a_{1}^{2 i} a_{2}^{2 j} b^{l}[M]$ with $2 i+4 j+l=4 m+1$, so $l$ must be odd. Then

$$
\begin{aligned}
a_{1}^{2 l} a_{2}^{2 \jmath} b^{l}[M] & =b\left(a_{1}^{2 i} a_{2}^{2 j} b^{l-1}\right)[M]=w_{1}\left(a_{1}^{2 i} a_{2}^{2 j} b^{l-1}\right)[M] \\
& =\operatorname{Sq}^{1}\left(a_{1}^{2 i} a_{2}^{2 j} b^{l-1}\right)[M]=0
\end{aligned}
$$

since $a_{1}^{2 i} a_{2}^{2 J} b^{l-1}$ is a square.

\section{REFERENCES}

[1] V. M. Buhštaber, Topological Applications of the theory of two-valued formal groups, Math. USSR Izvestija, 12 No. 1, (1978), 125-177.

[2] R. S. Clark and D. S. Goel, Almost tangent manifolds of second order, Tôhoku Math. J., 24 (1972), 79-92.

[3] P. E. Conner and E. E. Floyd, Fibring within a cobordism class, Michigan Math. J., 14 (1967), 289-303.

[4] H. Epiopoulos, Structures $r$-tangentes sur les variétés différentiable, C. R. Acad. Sci. Paris Ser A-B, 263 (1966), A 413-416.

[5] E. E. Floyd, Stiefel-Whitney numbers of quaternionic and related manifolds, Trans. Amer. Math. Soc., 155 (1971), 77-94.

[6] J. L. Rosendo and P. M. Gadea, Almost tangent structures of order $k$ on spheres, Analele stiintifice ale Universitatătii “Al. I. Cuza” din Iasi Tomul XXIII, S. I. a, f. 2, 1977, 281-286.

[7] R. E. Stong, On fibering of cobordism classes, Trans. Amer. Math. Soc., 178 (1973), 431-447.

[8] Subbundles of the tangent bundle, Trans. Amer. Math. Soc., 200 (1974), 185-197.

[9] _ Manifolds which immerse in small codimension, to appear in Illinois J. Math.

[10] Comments on Yoshida's manifolds, preprint.

[11] T. Yoshida, Wu classes and unoriented bordism classes of certain manifolds, Hiroshima Math. J., 10 (1980), 567-596.

Received April 13, 1983.

UNIVERSITY OF OKLAHOMA

NORMAN, OK 73019 



\title{
PACIFIC JOURNAL OF MATHEMATICS EDITORS
}

\author{
Donald BABBITt (Managing Editor) \\ University of California \\ Los Angeles, CA 90024 \\ J. DugunduI \\ University of Southern California \\ Los Angeles, CA 90089-1113 \\ R. FINN \\ Stanford University \\ Stanford, CA 94305 \\ HermanN FlaschKa \\ University of Arizona \\ Tucson, AŻ 85721
}

C. C. Moore

University of California

Berkeley, CA 94720

ARTHUR Ogus

University of California

Berkeley, CA 94720

Hugo Rossi

University of Utah

Salt Lake City, UT 84112

H. SAMELSON

Stanford University

Stanford, CA 94305

ASSOCIATE EDITORS
R. ARENS
E. F. BECKENBACH
B. H. NEUMANN
F. WOLF
K. YoSHIDA (1906-1982)

\section{SUPPORTING INSTITUTIONS}

$\begin{array}{ll}\text { UNIVERSITY OF ARIZONA } & \text { UNIVERSITY OF OREGON } \\ \text { UNIVERSITY OF BRITISH COLUMBIA } & \text { UNIVERSITY OF SOUTHERN CALIFORNIA } \\ \text { CALIFORNIA INSTITUTE OF TECHNOLOGY } & \text { STANFORD UNIVERSITY } \\ \text { UNIVERSITY OF CALIFORNIA } & \text { UNIVERSITY OF HAWAII } \\ \text { MONTANA STATE UNIVERSITY } & \text { UNIVERSITY OF TOKYO } \\ \text { UNIVERSITY OF NEVADA, RENO } & \text { UNIVERSITY OF UTAH } \\ \text { NEW MEXICO STATE UNIVERSITY } & \text { WASHINGTON STATE UNIVERSITY } \\ \text { OREGON STATE UNIVERSITY } & \text { UNIVERSITY OF WASHINGTON }\end{array}$

The Supporting Institutions listed above contribute to the cost of publication of this Journal, but they are not owners or publishers and have no responsibility for its content or policies.

Mathematical papers intended for publication in the Pacific Journal of Mathematics should be in typed form or offset-reproduced (not dittoed), double spaced with large margins. Please do not use built up fractions in the text of the manuscript. However, you may use them in the displayed equations. Underline Greek letters in red, German in green, and script in blue. The first paragraph must be capable of being used separately as a synopsis of the entire paper. In particular it should contain no bibliographic references. Please propose a heading for the odd numbered pages of less than 35 characters. Manuscripts, in triplicate, may be sent to any one of the editors. Please classify according to the scheme of Math. Reviews, Index to Vol. 39. Supply name and address of author to whom proofs should be sent. All other communications should be addressed to the managing editor, or Elaine Barth, University of California, Los Angeles, California 90024.

There are page-charges associated with articles appearing in the Pacific Journal of Mathematics. These charges are expected to be paid by the author's University, Government Agency or Company. If the author or authors do not have access to such Institutional support these charges are waived. Single authors will receive 50 free reprints; joint authors will receive a total of 100 free reprints. Additional copies may be obtained at cost in multiples of 50 .

The Pacific Journal of Mathematics is issued monthly as of January 1966. Regular subscription rate: $\$ 190.00$ a year (5 Vols., 10 issues). Special rate: $\$ 66.00$ a year to individual members of supporting institutions.

Subscriptions, orders for numbers issued in the last three calendar years, and changes of address should be sent to Pacific Journal of Mathematics, P.O. Box 969, Carmel Valley, CA 93924, U.S.A. Old back numbers obtainable from Kraus Periodicals Co., Route 100, Millwood, NY 10546.

The Pacific Journal of Mathematics at P.O. Box 969, Carmel Valley, CA 93924 (ISSN 0030-8730) publishes 5 volumes per year. Application to mail at Second-class postage rates is pending at Carmel Valley, California, and additional mailing offices. Postmaster: Send address changes to Pacific Journal of Mathematics, P.O. Box 969, Carmel Valley, CA 93924.

PUBLISHED BY PACIFIC JOURNAL OF MATHEMATICS, A NON-PROFIT CORPORATION

Copyright $@ 1984$ by Pacific Journal of Mathematics 


\section{Pacific Journal of Mathematics}

\section{Vol. 115, No. $2 \quad$ October, 1984}

Ersan Akyildiz, Gysin homomorphism and Schubert calculus ...........257

Marilyn Breen, Clear visibility and unions of two starshaped sets in the

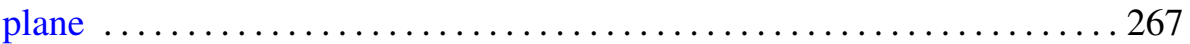

Robert F. Brown, Retraction methods in Nielsen fixed point theory . ......277

Herbert Busemann and Bhalchandra B. Phadke, A general version of Beltrami's theorem in the large ............................... 299

Gerald Arthur Edgar and Robert Francis Wheeler, Topological properties of Banach spaces ............................... 317

Yaakov Friedman and Bernard Russo, Conditional expectation without

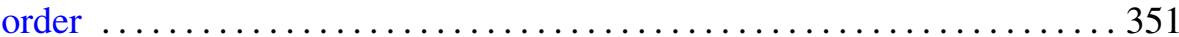

Robert Allen Goggins, Cobordism of manifolds with strong almost tangent

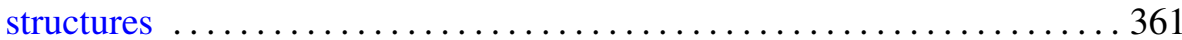

Mike Hoffman, Noncoincidence index of manifolds . . . . . . . . . . . . . 373

William H. Julian, $\varepsilon$-continuity and monotone operations $\ldots \ldots \ldots \ldots 385$

Gerasimos E. Ladas, Y. G. Sficas and I. P. Stavroulakis, Nonoscillatory

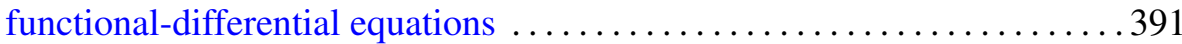

Arnold William Miller and Karel Libor Prikry, When the continuum has

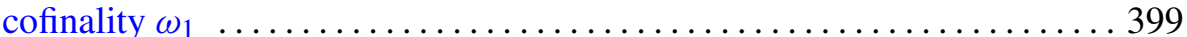

Jean-Leah Mohrherr, Density of a final segment of the truth-table

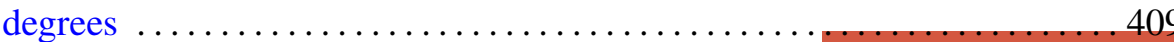

Carl Norman Mutchler, The flat Cauchy problem for radially hyperbolic operators from a characteristic manifold of high codimension ...

Kenji Nakagawa, On the orders of automorphisms of a closed Riemann surface

W. Ricker, Representation of vector-valued functions by Laplace transforms

Jorge Donato Samur, On semigroups of convolution operators in Hilbert

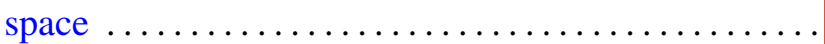

Joseph Gail Stampfli, One-dimensional perturbations of operators 481 Andrew George Earnest and John Sollion Hsia, Correction to: "Spinor norms of local integral rotations. II" 